\title{
MICRO PULSED RADIO-FREQUENCY ELECTROPORATION CHIPS
}

\author{
Huiqi He $^{\mathrm{a}, \mathrm{c}}$, Donald C. Chang ${ }^{\mathrm{b}}$ and Yi-Kuen Lee ${ }^{* a, c}$ \\ ${ }^{\mathrm{a}}$ Bioengineering Graduate Program, ${ }^{\mathrm{b}}$ Department of Biology \\ ${ }^{\mathrm{C}}$ Department of Mechanical Engineering \\ Hong Kong University of Science and Technology \\ Clear Water Bay, Kowloon, Hong Kong SAR, China
}

\begin{abstract}
Electroporation (EP) is one of the most important physical methods in biotechnology, which employs electrical pulses to transiently permeabilize cell membranes. In this study, a new micro pulsed radio-frequency electroporation cell ( $\mu$ PREP) chip was fabricated using a lift-off technique and SU-8 photolithography. The biological tests were carried out using three different plant protoplasts (cabbage, spinach and oil rape) on the micro EP chip and a pulsed RF electric field was applied to the microchip. The variations of fluorescent intensity and cell viability as functions of the electric pulse amplitude and duration time during the electroporation process were studied in detail at the single-cell level. Using such chip design and test method, one can easily optimize the efficiency and cell viability. Also, a large amount of statistical data can be quickly obtained. Finally, results of this parametric study were presented in the "phase diagram”, from which the critical electric field for inducing single-cell electroporation under different conditions can be clearly determined.
\end{abstract}

Keywords: micro electroporation, protoplast cells, SU-8, propidium iodide, membrane permeability

Bioelectrochemistry Vol. 68, No.1, pp. 89-97, 2006

DOI: 10.1016/j.bioelechem.2005.05.005

\footnotetext{
* Author to whom correspondence should be addressed. Department of Mechanical Engineering, HKUST, Clear Water Bay, Kowloon, Hong Kong SAR, China E-mail: meyklee@ust.hk. Tel: +852 2358 8663, Fax: +852 23581543
} 


\section{Introduction}

Electroporation (EP) is a widely used technique for the delivery of drugs and macromolecules such as DNA, to cells and tissues [1]. It uses an electric field pulse to induce nanometer-sized pore formation in the cell membrane such that the membrane is highly permeable to exogenous molecules presented in the surrounding media [2]. In comparison to the other methods of gene transfer such as lipofectamine treatment, electroporation has many advantages. It does not alter the biological structure and/or function of the target cells as electroporation is a noninvasive, non-chemical method.

Micro Electro-mechanical System (MEMS) based electroporation devices are attracting great attention nowadays. It reduces the potential risk of cells being damaged by high voltage and provides a better transfection efficiency and cell viability compared with conventional electroporation systems [3]. It requires a smaller number of cells and genes, and a much simpler cell preparation process. Furthermore, micro electroporation devices can control the electroporation at single-cell levels, which can not be achieved in traditional electroporation systems such as the Gene Pulser $^{\mathrm{TM}}$ (Bio-Rad Laboratories, CA, USA).

Since Murakami et al. used a micromachined electroporation system to electroporate fish cells in 1994 [4], many MEMS-based electroporation devices have been developed [510]. Unlike the traditional electroporation instruments, which can only electroporate the cells in bulk, the micro EP system can realize the control of electroporation process in individual cells [5]. Some even can detect various types of cells, capture their impedance transients and then electroporate them with the appropriate pulse parameter [6]. The idea of using microelectrode arrays was presented as a perspective method for biosensing and membrane electroporation in biomedical research and new clinical electrotherapies [7]. With those flowthrough micro electroporation chips, it was possible to perform continuous electroporation on the continuous flow of cells with a high transfection rate and a high survival rate [8]. The 
special design of multi-holed stainless steel electrodes was also utilized to eliminate the nonuniform flow rate of the cell suspension in this kind of EP chips [9]. For the selective electroporating of target cells, Lepioufle et al. also adopted the immobilization technology to manipulate the cells in the microarray with an antibody-antigen reaction, which guaranteed that only the diseased-cell were inserted with the therapeutic gene [10].

In addition to animal cell research, gene transformation is also highly important in plant biotechnology. Breeding techniques involving gene transfer through sexual and vegetative propagation are well established; the aim being to introduce genetic diversity into plant populations, to select superior plants carrying genes for desired traits and to maintain the range of plant varieties. The application of these conventional or classical techniques has produced significant achievements in the yield improvement of major food crops. However, this takes a long time, usually 6-8 years to produce a new variety of wheat or rice by sexual propagation [11]. It is very important to use the genetic engineering techniques to investigate plant cells for increasing farming productivity and insect resistance, etc.

In this work, a new micro pulsed $\underline{\mathrm{R} F}$ electroporation ( $\mu \mathrm{PREP}$ ) chip with multiple inputs was designed and fabricated for the parametric study of electroporation at the singlecell level on plant protoplasts. The surface treatment for immobilization of cells in the microarray can be easily carried out and a large number of cells can be electroporated at the same time with different electric pulse parameters. Numerical simulations are performed to study the non-uniform electric field distribution in the chip, and the quantitative analysis of cell membrane permeability and viability are described in detail.

\section{Experimental}

\subsection{Device Fabrication}


As schematically shown in Fig. 1, a $\mu$ PREP chip containing an array of $20 \times 24$ micro cell chambers was fabricated on a 4-inch glass wafer (Pyrex 7740, Dow Corning, USA) by two-mask MEMS technology. The multiple-input gold electrodes were defined during the first mask using the standard lift-off process, while the second mask formed the cell chamber using SU-8 photolithography (Microchem Corp., USA). Twelve pairs of commonly grounded electrodes with different inputs were fabricated on the glass wafer. With such a design, 12 groups of results with different electric pulse amplitudes and duration times can be obtained on a single chip at the same time. This chip can dramatically reduce the testing time by about one order of magnitude in comparison with our previous micro electroporation chip [12]. Each segmented electrode was made of $20 \mathrm{~nm}$ thick sputtered titanium and $500 \mathrm{~nm}$ thick sputtered gold with a width of $30 \mu \mathrm{m}$, by using a CVC 601 sputtering system. The space between adjacent electrodes was $30 \mu \mathrm{m}$. As shown in Fig. 1(a), both the width and length of each cell chamber are $30 \mu \mathrm{m}$, which is similar to the cells' diameter, to ensure that a singlecell is trapped in each cell chamber. The depth of the SU-8 photo resist was also set at $30 \mu \mathrm{m}$. The finished wafer was then diced and packaged on a printed circuit board (PCB) using wirebonding technology. A $1 \mathrm{~mm}$ diameter hole was drilled on the PCB at the area of the chip, as shown in Fig.1(d), for optical detection under an inverted fluorescence microscope (Olympus IX70, Japan). PDMS (Sylgard 184, Dow Corning, USA), a biocompatible polymer material, was coated on the wire-bonded site and cured at $150{ }^{\circ} \mathrm{C}$ for 1 min to protect the wire from possible breakage during the cleaning process. Photographs of the die and packaged micro electroporation chip are shown in Fig. 1(b) and Fig. 1(c) respectively.

\section{Fig. 1.}

Fig. 2 illustrates the micro fabrication process flow for the $\mu$ PREP chip. The modified lift-off process was used to achieve a thick metal layer $(500 \mathrm{~nm})$ while avoiding the hazardous solvent process. To obtain enough undercut for the lift-off metallization 
application, LOL2000 (Shipley Co., MA, USA) was double-coated as the bottom layer at $1000 \mathrm{rpm}$ for $30 \mathrm{~s}$, which resulted in the thickness of $1 \mu \mathrm{m}$ (Fig. 2(b)). A layer of FH6800L (Fujifilm Arch Co., Japan) was then spin-coated at $4000 \mathrm{rpm}$ for $60 \mathrm{~s}$ on the LOL2000 double layer. After patterning the FH6800L, Ti/Au (20 nm/500 nm) layers were sputtered on the front side of the wafer. The patterned electrode was then formed using the lift-off method in an ultrasonic bath of acetone (Fig. 2(c)). The $30 \mu \mathrm{m}$ SU-8 2025 photoresist was spin-coated at $2500 \mathrm{rpm}$ for $30 \mathrm{~s}$. Before the soft bake, the substrate was placed level for $20 \mathrm{~min}$ to eliminate thickness variation in the SU-8 layer. The second mask, which defined the electrodes, was aligned and the wafer was exposed to UV light. After 10 min resting time, the wafer was baked at $70^{\circ} \mathrm{C}$ for $1 \mathrm{~min}$, and at $90^{\circ} \mathrm{C}$ for $3 \mathrm{~min}$ and used for photoresist development.

\section{Fig. 2}

Before applying an electric field pulse to the $\mu$ PREP chip, the cells should be loaded on the chip and filled in each cell chamber for subsequent electroporation. However, since the surface of cross-linked SU-8 on the chip was strongly hydrophobic due to a contact angle of about 102 degrees (measured by a contact angle meter (Digidrop, GBX Co., France)), cells were difficult to be filled in without any surface treatment. To solve this problem, we introduced the oxygen plasma treatment (PDC-002, Harrick Sci. Corp., USA) in our experiment to change the surface condition from hydrophobic to hydrophilic with a stable contact angle of about 20 degrees. As shown in Fig. 3, the hydrophilic surface property can efficiently enhance the cell loading performance prior to the experimental tests. Since the size of chambers is $30 \mu \mathrm{m}$, similar to the average diameter of the plant protoplasts, each chamber can only contain one cell and such surface treatment can provide $80 \%$ of cell chambers being successfully filled with cells in the array of micro cell chambers on the chip. Compared to the complicated bio-immobilization deposition process [10], the proposed 
physical process has the advantages of easy manipulation and cleaning. In addition, all the materials used are biocompatible and the fabrication process is also IC-process compatible. Thus, this chip could be used for large-scale gene transfection and drug screening.

Fig. 3

\subsection{Experimental Setup}

As illustrated in Fig. 4(a), the pulsed radio-frequency (RF) electric field with adjustable frequency, amplitude and duration time was applied to the $\mu$ PREP chip using a Labview program and the PCI 6110 DAQ card (National Instrument, TX, USA). The main advantage of a pulsed RF electric field is that it can counterbalance the cells' size effect with an opposite effect of cell relaxation $[13,14]$. As a result, the viability and permeability of the electroporation can be improved comparing with the traditional DC pulse or exponential decay pulse $[13,14,15]$. The DAQ card has two 16-bit analog outputs with a maximum voltage of $10 \mathrm{~V}$ and a maximum frequency of $4 \mathrm{MHz}$. The operational-amplifier (LF412C, Motorola) based circuit, shown in Fig. 4(b), was used to divide one channel output into 6 RF signals with different pulse amplitudes, which are directly proportional to the original output as follows:

$U_{n}=U \times \frac{R w_{n}}{R}(n=1,2 \ldots 6)$

where $U_{\mathrm{n}}$ is the amplitude of the sine wave for each RF signal, $U$ is the amplitude of the voltage output from the DAQ card, $R$ and $R w_{\mathrm{n}}$ are the resistances as shown in Fig. 4(b). Additional RC circuits, which consist of $R_{f}$ and $C_{f}$, in the output stage were used to filter the high frequency noises at a cutoff frequency of $40 \mathrm{kHz}$ (here we choose $R_{f}$ and $C_{f}$ values of $680 \mathrm{pF}$ and $560 \Omega$ respectively). The electroporating pulse used in this study was $1-10 \mathrm{~ms}$ wide, DC-shifted RF pulse, which oscillated at $30 \mathrm{kHz}$ with an amplitude between $1 \mathrm{kV} / \mathrm{cm}$ and $3 \mathrm{kV} / \mathrm{cm}$.

\section{Fig. 4}


During the testing, an Olympus IX70 inverted fluorescent microscope and an QImaging Retiga 1300C thermoelectric cooled digital CCD camera (Burnaby, B.C., Canada) were used for in situ visualization of the cells' response. The cells on the microchips were illuminated by a 100 Watt mercury lamp with a set of fluorescence filters: excitation by a 515 $\mathrm{nm}$ to $565 \mathrm{~nm}$ bandpass filter and the emission by a $550 \mathrm{~nm}$ to $655 \mathrm{~nm}$ bandpass filter. Sets of images, including image and background, were acquired by the image capture card. Image enhancement programs including histogram stretching, edge finding and thresholding were developed in Matlab ${ }^{\mathrm{TM}}$ (Mathworks Inc., MA, USA) to enhance the background-subtracted images and to automatically find the intracellular areas. In order to quantify the effects of pulse duration time $\left(t_{p}\right)$ and the applied voltage $\left(V_{a}\right)$ on transport of fluorescent dye, the average fluorescent intensity $\bar{I}$ of each cell was determined.

\subsection{Electric field simulation}

In order to understand the micro EP process, numerical simulations were performed, using a commercial code (CFD-ACE+, ESI CFD Inc., AL, USA), to study the electric field distribution in the $\mu$ PREP chip. Based upon the exact size of the chip, the typical electric field distribution in the vicinity of electrodes was simulated as shown in Fig. 5. The electric field distribution across the cell chamber is non-uniform. Most of the high electric field is close to the micro electroporation chip surface and concentrated between the two electrodes, while in other areas the electric field is much weaker. This is in contrast to the conventional curvette where the electric field is uniformly distributed across the volume between the two parallel planar electrodes.

\section{Fig. 5}

The average electric field within the chip can then be obtained by averaging the nonuniform electric field distribution for each data point within the electrodes area. Therefore, the externally applied voltage can be converted into the average electric field $\left(E_{a v g}=\alpha V_{a} / L_{\mathrm{e}}\right)$ 
via a correction factor ( $\alpha=0.630)$ calculated from the numerical simulation. Note that $L_{e}$ is the distance between the two adjacent electrodes. Although the simulation model did not include the cell, the correction factor calculated from the electric field simulation can still be useful for design of the $\mu$ PREP chip.

\subsection{Cell Suspension Preparation}

Plant protoplasts were isolated by enzymatic digestion of various tissues and the cell walls are removed [16]. Leaf slices of cabbage (Brassica pekinensis Rupr.), spinach and oil rape (Brassica napus) were prepared and cut into segments of $0.5-1 \mathrm{~mm}$. They were then incubated for three hours at room temperature (about $25^{\circ} \mathrm{C}$ ) in the digestion medium whose $\mathrm{pH}$ value was adjusted to 5.5 with $\mathrm{KOH}$ prior to the adding of enzymes. The digestion medium comprised 500 mM D-Sorbitol (Sigma, S-1876), 1 mM CaCl 2 (Sigma, 23922-4), 5 mM MES (Sigma, M-8250), Cellulase Trichoderma viride (Sigma, C-9422, 400U), and Pectinase from Rhizopus (Sigma, P-2401, 300U). After the incubation, the digestion medium was carefully removed and discarded. The plant tissue was then washed using $5 \mathrm{~mL}$ wash medium comprising $500 \mathrm{mM}$ D-Sorbitol, $1 \mathrm{mM} \mathrm{CaCl}_{2}$, and $5 \mathrm{mM}$ MES. The $\mathrm{pH}$ value of the wash medium was adjusted to 6 with $\mathrm{KOH}$. The tissue was finally collected on a Millipore filter with nylon mesh, of which the pore diameter was $60 \mu \mathrm{m}$, and the vascular tissue and undigested material were removed. To quantitatively detect the results of electroporation and the fluorescent dye, propidium iodide (PI) (Sigma, P-4170, excitation/emission peak (DNAbind) $=535 \mathrm{~nm} / 617 \mathrm{~nm}$ ), which is a membrane-impermeable nucleic acid intercalator with molecular weight 668.4, was used in the experiments. Since PI dye is a non-autofluorescent dye, it can only become fluorescent after being attached to nucleic acids. Therefore, without background fluorescence, only the dye molecules inside the cells and bound to the DNA/RNA are able to emit light under UV light excitation. Thus, the contrast is high between the cells uploaded with dye molecules and the background. This ensures that good- 
quality fluorescent images are obtained. In the experiments, PI dye was dissolved in the cell suspension prior to the electroporation in order to achieve the desired concentration of 15 $\mu \mathrm{g} / \mathrm{mL}$.

\section{Result and Discussion}

\subsection{Cell membrane permeability}

Permeabilization of plant protoplasts can be detected through the change of fluorescent intensity within the cells. However, influx of fluorescence dye can occur only for electric field and pulse duration time higher than their thresholds. The uptake of PI was quantified by the associated fluorescence intensity that reflects its free diffusion across the permeabilized parts of the cytoplasm membrane. Figs. 6(a)-(d) show the micrographs of the protoplast cells filled in the array of $20 \times 24$ micro cell chambers on a micro EP chip at different stages. The cell array under a fluorescent microscope before electroporation, shown in Fig. 6(a), remains dark due to the impermeability of the plasma membrane. After electric pulse treatment, PI dye diffuses into the cell through the nano scale pores induced by the electric field and binds with DNA/RNA, thus the cell becomes brighter as shown in Fig. 6(b). Fig. 6(c) shows the bright-field image of permeabilized cabbage cells. The nucleus can be clearly recognized in Fig. 6(d) since it is rich in DNA/RNA molecules.

Fig. 6

The density and the duration time of the pores on the surface of cells vary with the applied electric pulse strength and width. The fluorescent intensity variations of the cells as functions of respectively the pulse amplitude and pulse duration time during the

electroporation are shown in Fig. 7. It can be seen that varying the pulse amplitude and pulse duration time have similar effects on the fluorescent intensity. As shown in Fig. 7(a), the electroporation process can be divided into three stages: 
1. The un-electroporated stage, where the pulse amplitude is smaller than the critical voltage and the electric field is not strong enough to induce nanometer-sized pores formed on the cell membrane.

2. The electroporated stage, where the pulse amplitude is above the critical voltage and the dye molecules can diffuse into the cell through the pores. The critical voltages are different for different pulse duration times. In general, the critical voltage is lower for longer pulse duration time as it can be seen from Fig. 7. The critical electric fields (voltages) are $0.841 \mathrm{kV} / \mathrm{cm}(4 \mathrm{~V})$ and $1.261 \mathrm{kV} / \mathrm{cm}(6 \mathrm{~V})$ for pulse duration times of $6 \mathrm{~ms}$ and 2 ms respectively.

3. The saturation stage, where the fluorescent intensity becomes stable. Further increase of the voltage does not increase the membrane permeability any more. At this stage some cells can undergo lysis. The higher the voltage, the more the cells undergo lysis. In a practical application, for the purpose of achieving both high cell viability and permeability, a voltage close to the boundary of the linear and the saturation regions should be chosen.

Fig. 7

Fig. 7(c) shows the "phase diagram" as a function of electric field and pulse duration time, which delineates the boundary of the areas for cell lysis, electroporation and absence of electroporation. Electric field amplitude and pulse duration time can be traded off to a certain extent from this figure. For example, in order to induce electroporation, lower electric field amplitude needs longer pulse duration time to charge the cell membrane to reach the electroporation threshold membrane potential. On the other hand, if the electric field amplitude is high enough to achieved the breakdown transmembrane potential, it needs only short pulse duration time. Similarly, treatment of cells with intense electric field and short pulse duration time will lead to an unstable situation where the cell can not repair the damage 
and will be damaged (lysed), whereas cells can sustained a longer period of time if a mild electric field is applied. The phase diagram can be used as a guideline for electric parameter selection to improve the electroporation efficiency, and with our micro electroporation chip, a large amount of statistical data can be easily obtained to promptly build up this kind of phase diagram. The uptake of small fluorescence molecules by each cell as a function of pulse amplitude under different pulse duration time were fitted to the sigmoidal function, which has the same functional form as described in literature [17]. Furthermore, the electroporation area outlined in Fig.7 (c) matches reasonably with previous results [18] which used traditional electroporation instruments.

Although membrane electroporation is a ubiquitous phenomenon to all types of cells, the permeabilization varies significantly between different cell lines. Specific empirical protocols need to be developed for each cell line in order to achieve optimal results. In this study, 3 types of plant cells, i.e., Brassica pekinensis, spinach, and Brassica napus, were tested and the results are shown in Fig. 8(a)-(c) for different pulse duration times. As shown in Fig. 8(a), with the increase of applied voltage the fluorescent intensity of brassica napus cell increases more sharply compared to that of the spinach cell. The critical electric fields (voltages) for spinach and the brassica napus cells under the pulse duration time of $2 \mathrm{~ms}$ are $1.681 \mathrm{kV} / \mathrm{cm}$ $(8 \mathrm{~V})$ and $1.471 \mathrm{kV} / \mathrm{cm}(7 \mathrm{~V})$ respectively. As can be seen, the critical electric field and voltage for each cell can now easily be determined.

\section{Fig. 8}

\subsection{Cell viability}

The conventional method for detecting cell viability is by using the membraneimpermeable dye after the pores induced by electric treatment are resealed [19,20]. Because healthy cell membranes prevent those kinds of dye from entering cells, the dye molecule will not stain them. In contrast, since dead cells lose membrane integrity, they will be stained. 
Therefore, dead and living cells can be distinguished by examining their color or fluorescence under the microscope. In our experiment, cell viability was measured by adding electric pulses to the cells in the absence of dye. Viability was then assayed by PI dye staining half an hour after electroporation treatment instead of adding PI dye before electric pulse treatment as in the electroporation experiment. As shown in Fig. 9, the cell viability decreases with the increase of permeability (all data points were obtained from 4 experiments). In addition, higher electric pulse amplitude and longer duration time result in lower cell viability and higher permeability. It can also be seen that at the highest pulse amplitude, the survival rate is almost doubled for the duration time of 2 ms compared to that of $8 \mathrm{~ms}$. This clearly indicates that there is a trade-off between membrane permeability and viability. Therefore, the regions close to the cross point of the two curves can be an optimal choice for electroporation. In this study, the variations of cell viability in the experiment for the three different cells did not seem to be significant.

\section{Fig. 9}

\section{Conclusions}

A new micro pulsed radio-frequency electroporation cell ( $\mu$ PREP) chip has been successfully fabricated and tested with three different types of plant cells. For the first time, a detailed parametric study of the pulsed radio-frequency electric field was conducted for cell electroporation on microchips. Surface treatment of the microchip by oxygen plasma provided conditions for a simple and efficient cell-loading process. Different pulsed RF electroporation protocols can be easily programmed in the computer-controlled system. The experimental results demonstrate that high membrane permeability and viability can be achieved on the $\mu$ PREP chips. From this parametric study, the critical voltage to induce single-cell electroporation has been determined. In addition, it has been shown that the proposed cell-array design can easily provide a large amount of statistical data (more than 
one thousand data points), which is required for determining the critical voltage, by using digital image processing. The resultant phase diagram for the boundary of electroporation and cell lysis can be useful for the design of integrated micro TAS systems and high throughput analysis.

\section{Acknowledgements}

This work is supported by the Hong Kong Research Grant Council (HKUST 6017/02E and 6109/01M) and by the HKUST Institute of Microsystems (I2MS01/02.EG08. The authors would like to thank Prof. Yitshak Zohar at University of Arizona, Tucson, USA, Ms. Vivian C. Yu, Ms. Inez Tsui, Dr. Kathy Luo and Mr. Thomas W. H. Chau at HKUST for technical support. 


\section{References:}

[1] D. C. Chang, J. A. Saunders, B. M. Chassy, and A. E. Sowers, Overview of Electroporation and Electrofusion, in: D. C. Chang, B. M. Chassy, J. A. Saunders and A. E. Sowers, Guide to Electroporation and Electrofusion, Academic Press, San Diego, 1992, pp.1-6.

[2] D. C. Chang, T. S. Reese, Changes of membrane structure induced by electroporation as revealed by rapid-freezing electron microscopy, Biophys. J., 58 (1990), 1-12.

[3] Y. Huang, B. Rubinsky, Micro-Electroporation: Improving the efficiency and understanding of electrical permeabilization of cells, Biomedical Microdevices, 2 (1999), 145-150.

[4] Y. Murakami, K. Motohashi, K. Yano, K. Ikebukuro, K. Yokoyama, E. Tamiya, I. Karube, Micromachined electroporation system for transgenic fish, J. Biotechnol., 34 (1994), 35-42.

[5] Y. Huang, B. Rubinsky, Microfabricated electroporation chip for single cell membrane permeabilization, Sens. Actuators, A, 89 (2001), 242- 249.

[6] A. Sarkar, B. Mitra, A. Shastry, S. Wadia, R. Mulherkar, R. Lal, A low voltage single cell electroporator with a microfabricated sense-porate aperture, IEEE MEMS'04, Maastricht, Netherlands, January 25-29, 2004, 375-378.

[7] E. Neumann, K. Tonsing, P. Siemens, Perspectives for microelectrode arrays for biosening and membrane electroporation, Bioelectrochemistry, 51 (2000), 125-132.

[8] Y.-C. Lin, M.-Y. Huang, M. Li, Observation of extremely low transmembrane potential of cells in electroporation using microchips. MicroTAS’02, Nara, Japan, November 3-7, 2002, 847-849.

[9] M. Fukui, M. Yamada, N. Y. Lee, M. Seki, Microdevice for flow-through electroporation using multi-holed electrodes, MicroTas'03, Squaw Valley, California, USA, October 5-9, 2003, 331-334. 
[10] B. Lepioufle, P. Surbled, H. Nagi, K. S. Chun, Y. Murakami, E. Tamiya, H. Fujita, Living cells captured on a bio-microsystem devoted to DNA injection, Mater. Sci. Eng., C, 12 (2000), 77-81.

[11] H. S. Chawla, Introduction to plant biotechnology, Science publishers, Enfield, 2002.

[12] F. Yang, H. He, D. C. Chang, Y.-K. Lee, Parametric study of pulsed radio-frequency electroporation on microchips at the single-cell level, MicroTAS’04, Malmö, Sweden, September 26-30, 2004, Vol. 2, 79-81.

[13] D. C. Chang, Cell poration and cell fusion using an oscillating electric field, Biophys. J., 56 (1989), 641-652.

[14] D. C. Chang, P. Q. Gao, B. L. Maxwell, High efficiency gene transfection by electroporation using a radio-frequency electric field, Biochim. Biophys. Acta., 1092 (1991), 153-160.

[15] D. C. Chang, J. R. Hunt, Q. Zheng, P.-Q. Gao, Electroporation and electrofusion using a pulsed radio-frequency electric field, in: D. C. Chang, B. M. Chassy, J. A. Saunders and A. E. Sowers, Guide to Electroporation and Electrofusion, Academic Press, San Diego, 1992, pp.303-326.

[16] Plant protoplast preparation recipes, Serva Electrophoresis GmbH, Germany (http://www.serva.de/products/knowledge/081041.shtml).

[17] K. Nolkrantz, C. Farre, A. Brederlau, R. I. D. Karlsson, C. Brennan, P. S. Eriksson, S. G. Weber, M. Sandberg, O. Orwar, Electroporation of single cells and Tissues with an electrolyte-filled capillary, Anal. Chem. 73(2001), 4469-4477.

[18] S. B. Dev, D. P. Rabussay, G. Widera, G. A. Hofmann, Medical applications of electroporation, IEEE T Plasma Sci, 28 (2000), 206-223.

[19] Y.-C. Lin, C.-M. Jen, M.-Y. Huang, C.-Y. Wu, X.-Z. Lin, Electroporation microchips for continuous gene transfection, Sens. Actuators, B, 79 (2001), 137-143.

[20] M. Golzio, M. P. Rols, J. Teossie, In vitro and in vivo electric field-mediated permeabilization, gene transfer, and expression, Methods, 33 (2004), 126-135. 


\section{List of Figures}

Fig. 1. (a) 3D schematic diagram and cross section of the $\mu$ PREP chip; (b) photo image of a $\mu$ PREP chip die. The electrodes in vertical direction are used for multiple electric field input, while those in lateral direction are for detecting single cell's current during electroporation, which will be carried out in the future; (c) Photograph of a packaged device. Within the red circle is the $\mu$ PREP chip die, which is wire-bonded on the PCB board; (d) Schematic diagram of a packaged device for optical detection.

Fig. 2. Fabrication process flow diagram of a $\mu$ PREP chip.

Fig. 3. Comparison of the cell filling process in the array of micro cell chambers on a $\mu$ PREP chip with and without oxygen plasma treatment; (a) 1 min after loading the cells suspension to the chip without oxygen plasma treatment; (b) 1 min after loading the cells suspension in the chip that had 5 min oxygen plasma treatment (all scale bars: 30 $\mu \mathrm{m})$.

Fig. 4. (a) Experimental setup for the $\mu$ PREP chips; (b) The diagram of operational-amplifier based circuit used to divide one channel output into 6 RF signals with different pulse amplitudes.

Fig. 5. Simulation result of the electric field distribution on a cross section of the $\mu$ PREP chip.

Fig. 6. Optical micrographs of the protoplast cells filling in the array of $20 \times 24$ micro cell chambers on a $\mu$ PREP chip (only $14 \times 11$ can be shown due to limited field of view); (a) Before electroporation, the cells are dark under fluorescence mode; (b) Cells become white when successfully undergoing electroporation; (c) Bright-field image of permeabilized cabbage cells; (d) Fluorescent-field image of permeabilized cells (all scale bars: $30 \mu \mathrm{m})$.

Fig. 7. (a) Average fluorescent intensity as a function of pulse duration time ( $\left.t_{p}\right)$; (b) Average fluorescent intensity as a function of applied voltage $\left(V_{a}\right)$ on Brassica pekinensis mesophyll protoplast (each data point the average from 10 cells); (c) The phase diagram for electroporation and cell lysis.

Fig. 8. Average fluorescent intensity as a function of applied voltage on 3 kinds of plant cells (Brassica pekinensis, spinach, and Brassica napus); (a) $t_{p}=2 \mathrm{~ms}$; (b) $t_{p}=4 \mathrm{~ms}$; (c) $t_{p}=$ 6 ms (each data point the average from 10 cells).

Fig. 9. Effect of applied voltage and pulse duration time on cabbage cell membrane permeability and viability (continuous line : Avg. fluorescence intensity, dash line: cell viability); (a) $t_{p}=2 \mathrm{~ms}$; (b) $t_{p}=4 \mathrm{~ms}$; (c) $t_{p}=6 \mathrm{~ms}$; (d) $t_{p}=8 \mathrm{~ms}$. 


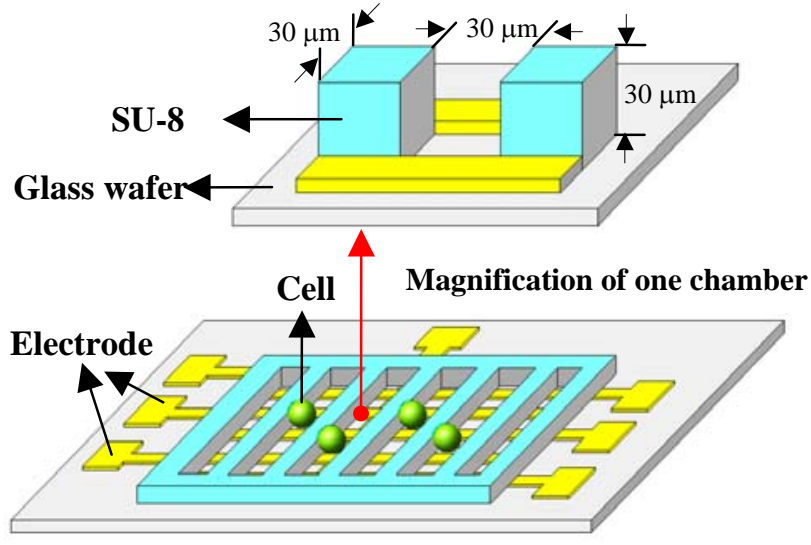

(a)

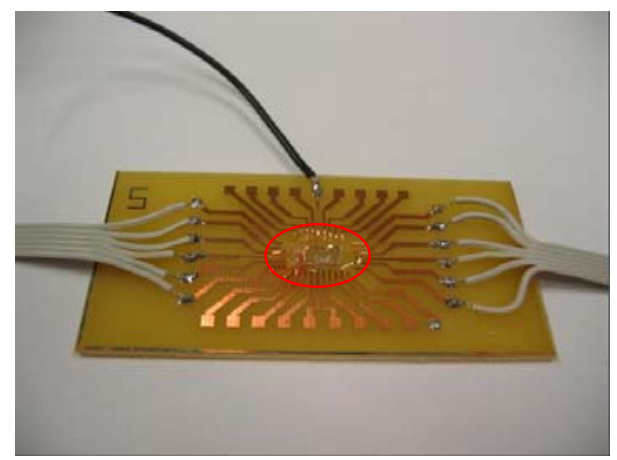

(c)

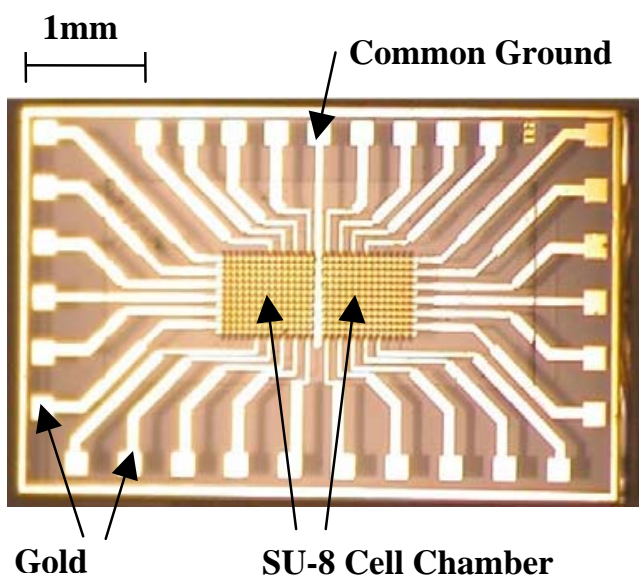

(b)

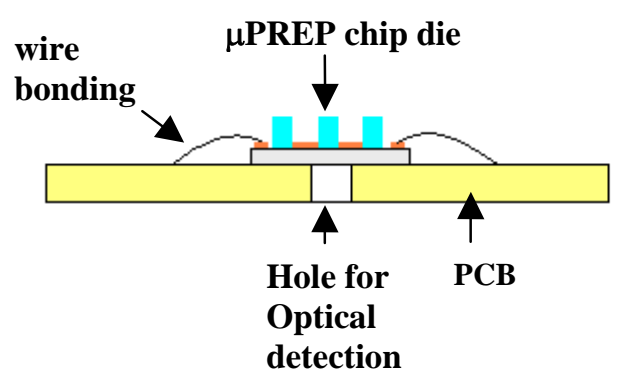

(d)

Fig. 1. (a) 3D schematic diagram and cross section of the $\mu$ PREP chip, (b) photo image of a $\mu$ PREP chip die. The electrodes in vertical direction are used for multiple electric field input, while those in lateral direction are for detecting single cell's current during electroporation which will be carried out in the future, (c) Photograph of a packaged device. Within the red circle is the $\mu$ PREP chip die which is wire-bonded on the PCB board, (d) Schematic diagram of a packaged device for optical detection. 
(a)

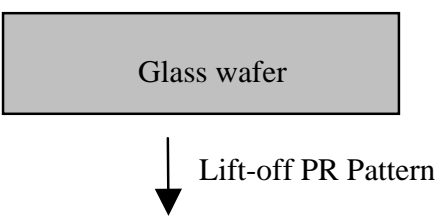

(b) $\mathbf{N} \quad \mathbf{N} \mathrm{N}$

Glass wafer

Sputter and Lift-off

(c)
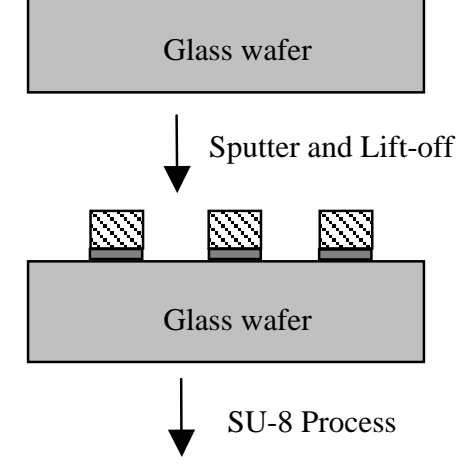

(d)

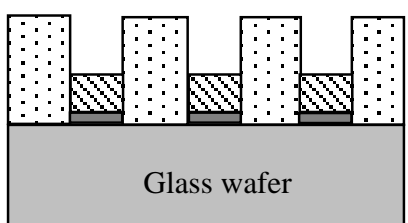

$\mathbf{D V}$ Lift-off PR Ti $\$$ Au $: \because:$ SU-8

Fig. 2. Fabrication process flow diagram of a $\mu$ PREP chip. 

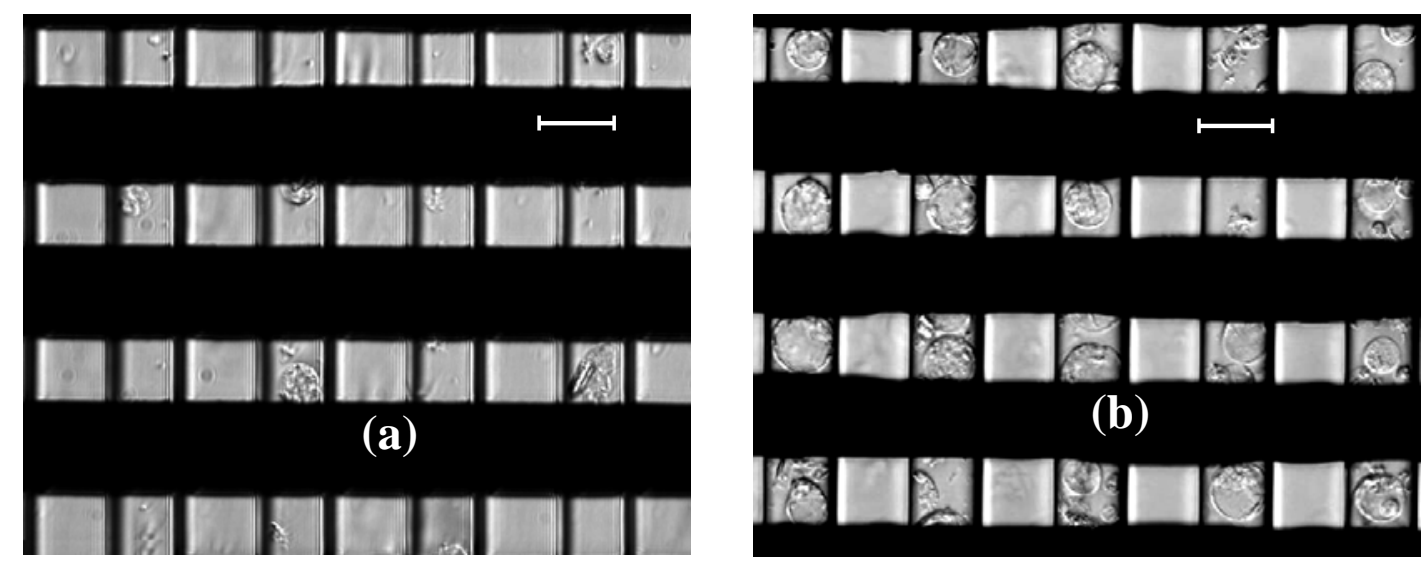

Fig. 3. Comparison of the cell filling process in the array of micro cell chambers on a $\mu$ PREP chip with and without oxygen plasma treatment; (a) 1 min after loading the cells suspension to the chip without oxygen plasma treatment; (b) 1 min after loading the cells suspension in the chip that had 5 min oxygen plasma treatment (all scale bars: $30 \mu \mathrm{m}$ ). 


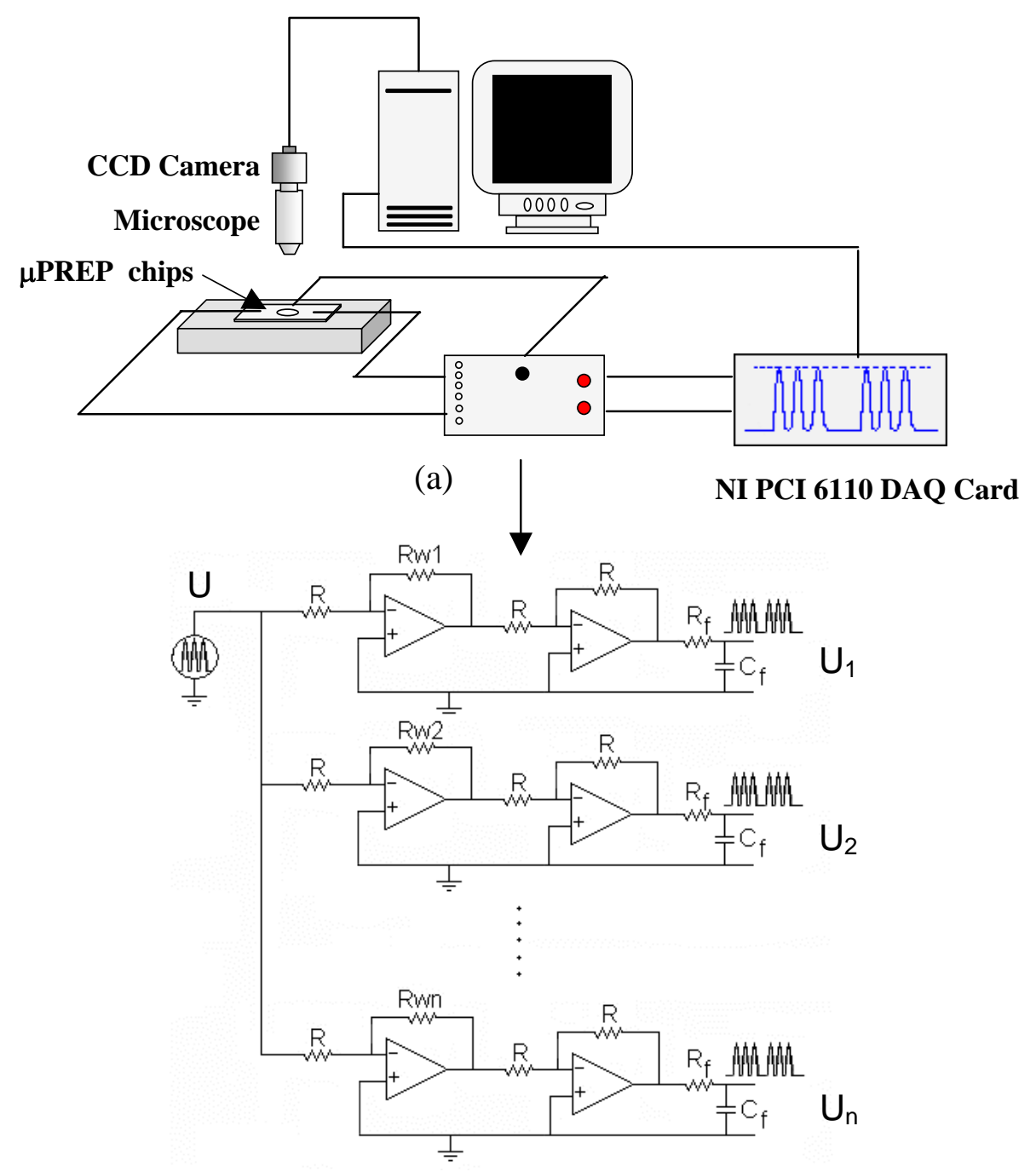

(b)

Fig. 4. (a) Experimental setup for the $\mu$ PREP chips; (b) The diagram of operational-amplifier based circuit used to divide one channel output into 6 RF signals with different pulse amplitudes. 


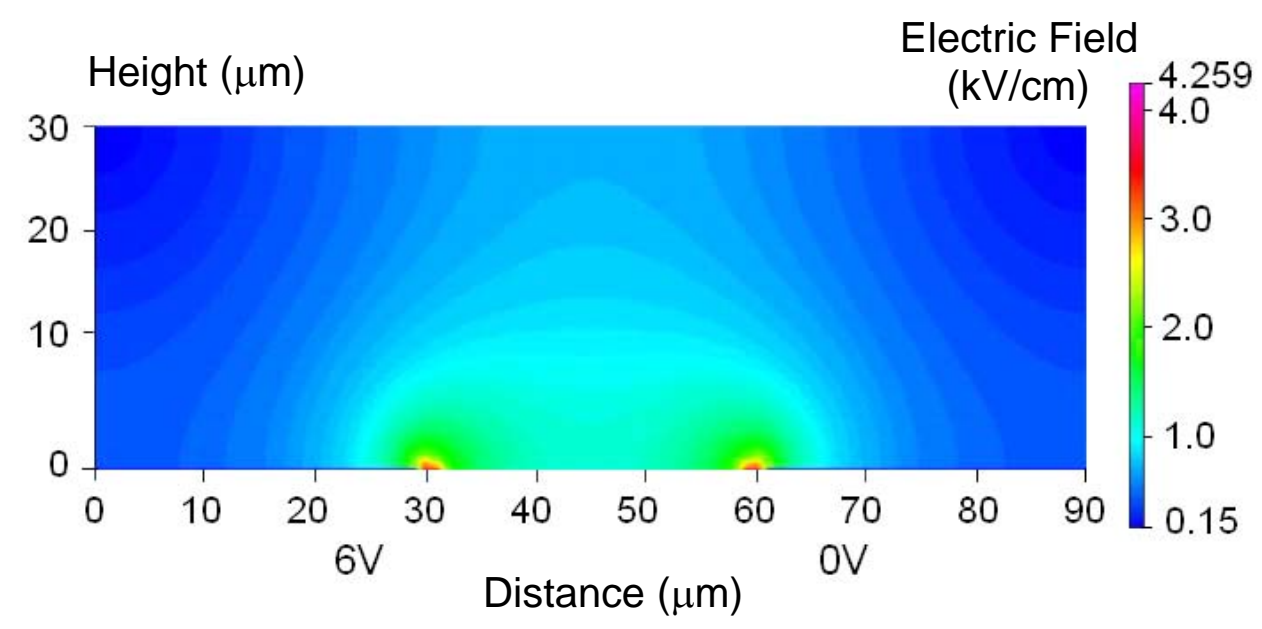

Fig. 5. Simulation result of the electric field distribution on a cross section of the $\mu$ PREP Chip. 

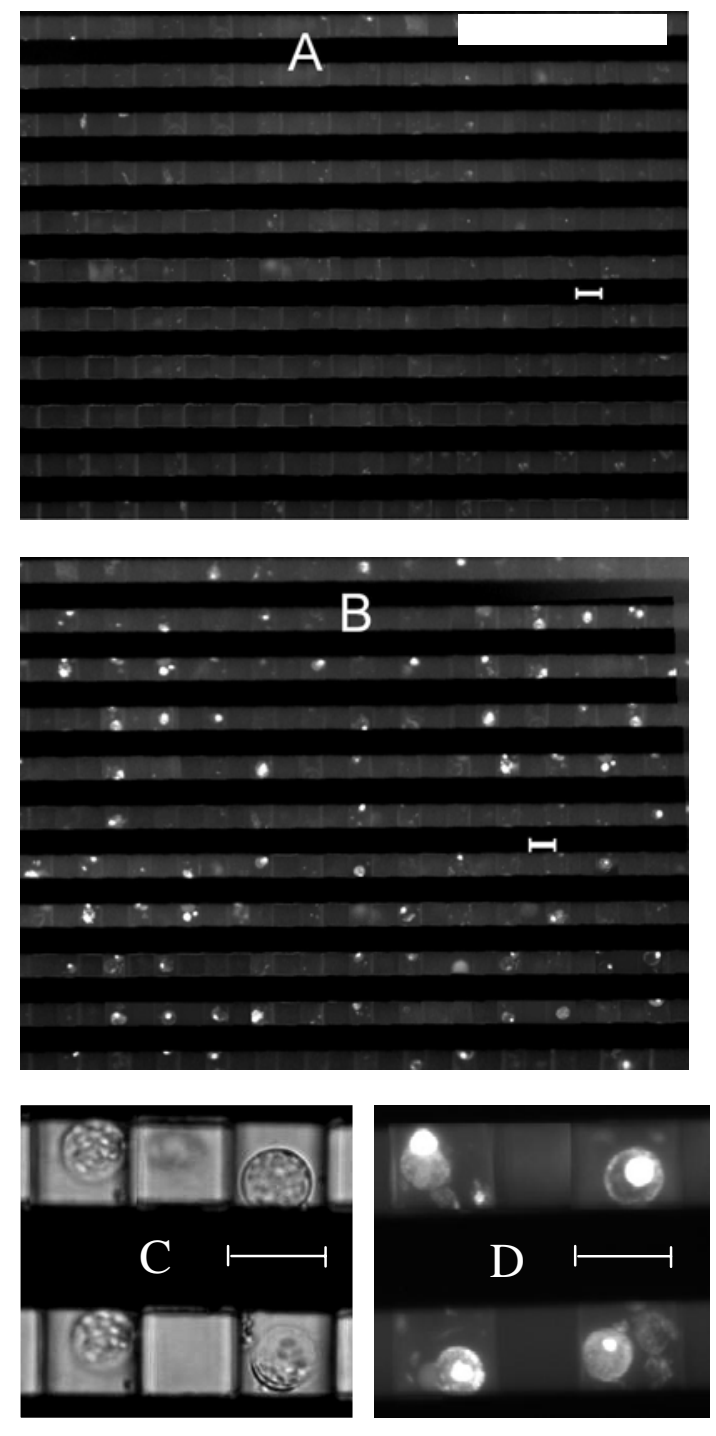

Fig. 6. Optical micrographs of the protoplast cells filling in the array of $20 \times 24$ micro cell chambers on a $\mu$ PREP chip (only $14 \times 11$ can be shown due to limited field of view), (a) Before electroporation, the cells are dark under fluorescence mode; (b) Cells become white when successfully undergoing electroporation; (c) Bright-field image of permeabilized cabbage cells; (d) Fluorescent-field image of permeabilized cells (all scale bars: $30 \mu \mathrm{m}$ ). 


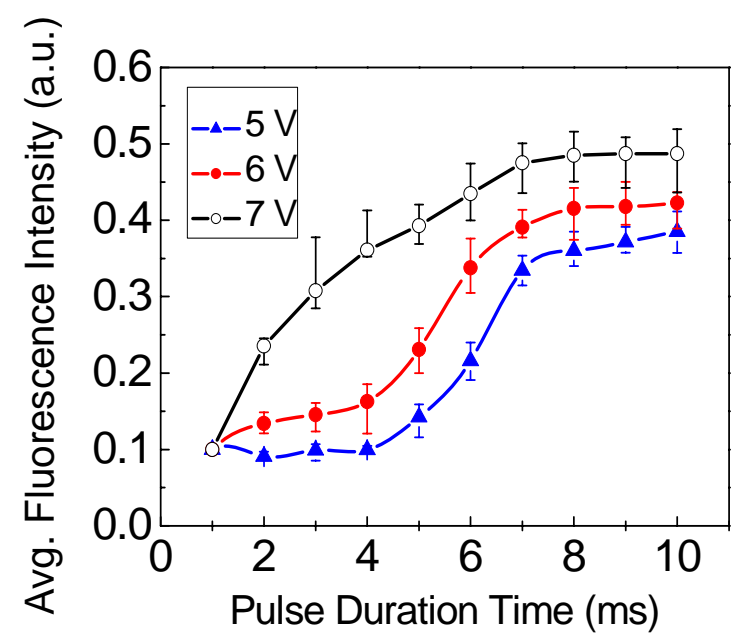

(a)

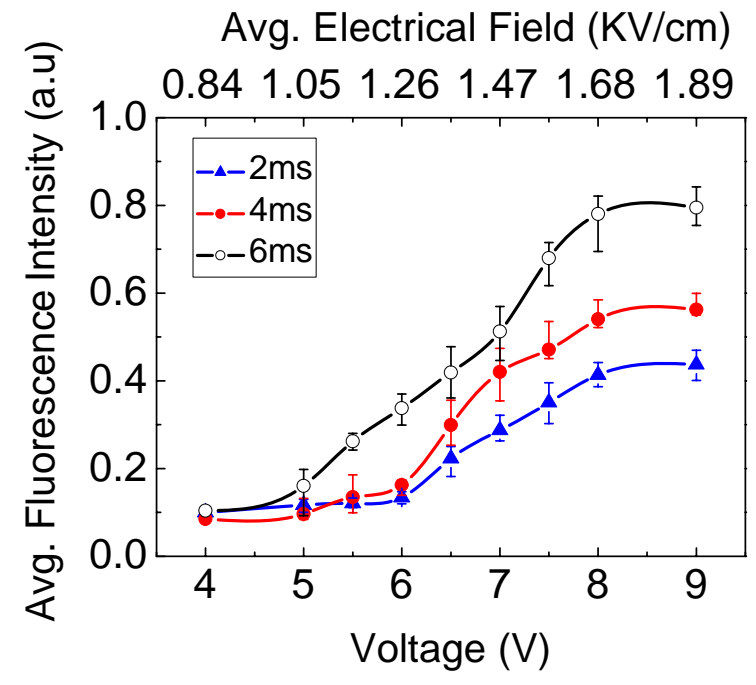

(b)

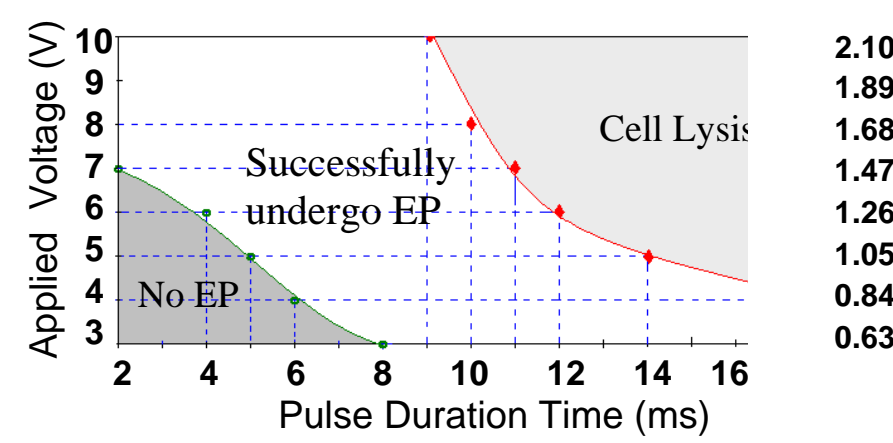

(c)

Fig. 7. (a) Average fluorescent intensity as a function of pulse duration time ( $\left.t_{p}\right)$, (b) Average fluorescent intensity as a function of applied voltage $\left(V_{a}\right)$ on Brassica pekinensis mesophyll protoplast (each data point the average from 10 cells); (c) The phase diagram for electroporation and cell lysis. 


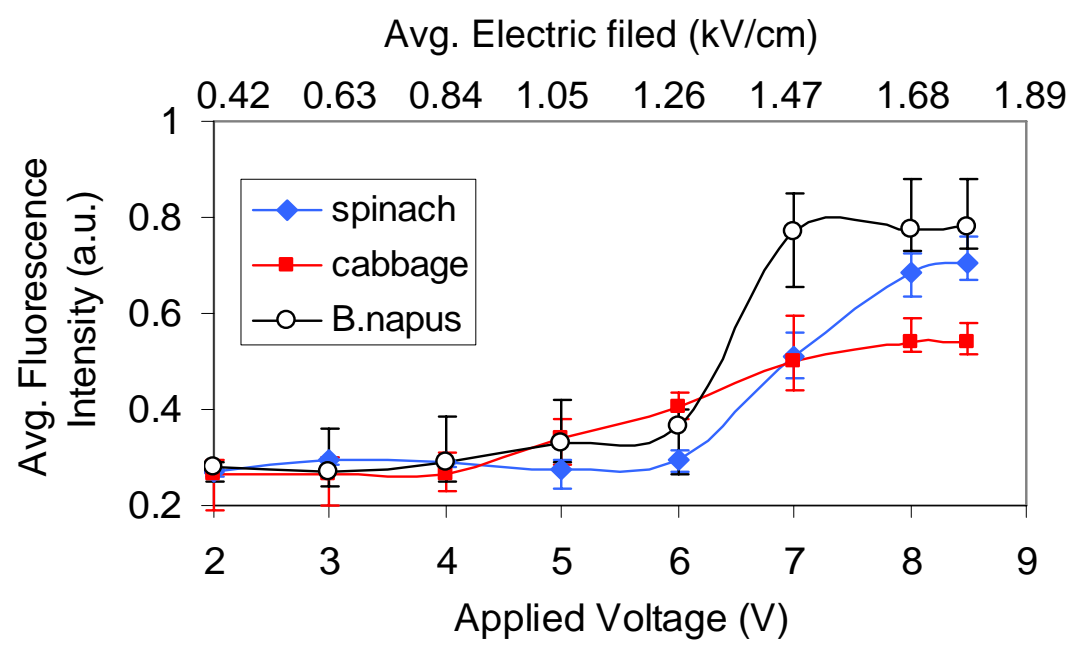

(a)

Avg. Electric filed $(\mathrm{kV} / \mathrm{cm})$

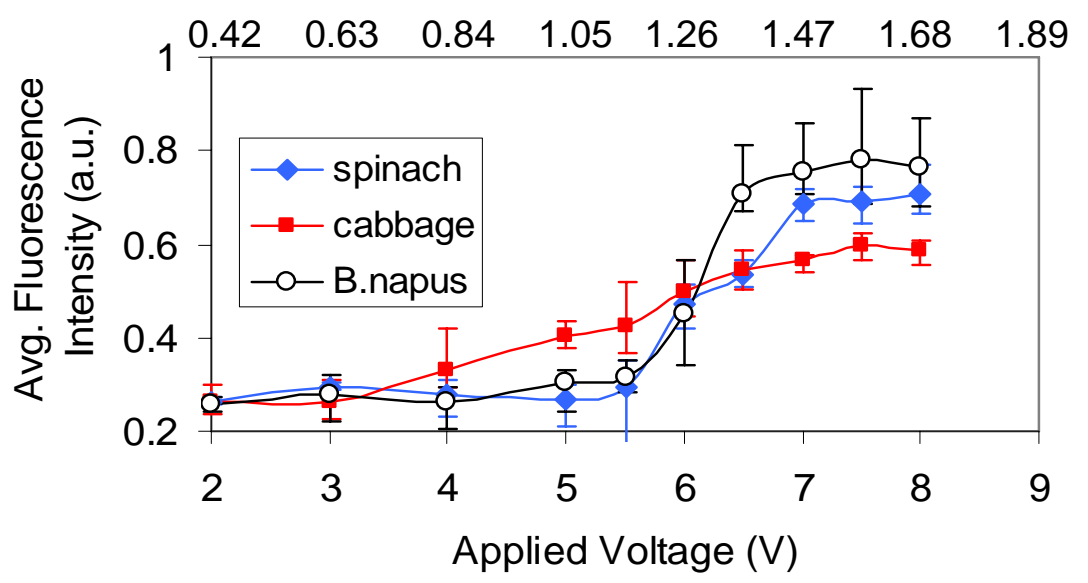

(b)

Avg. Electric filed $(\mathrm{kV} / \mathrm{cm})$

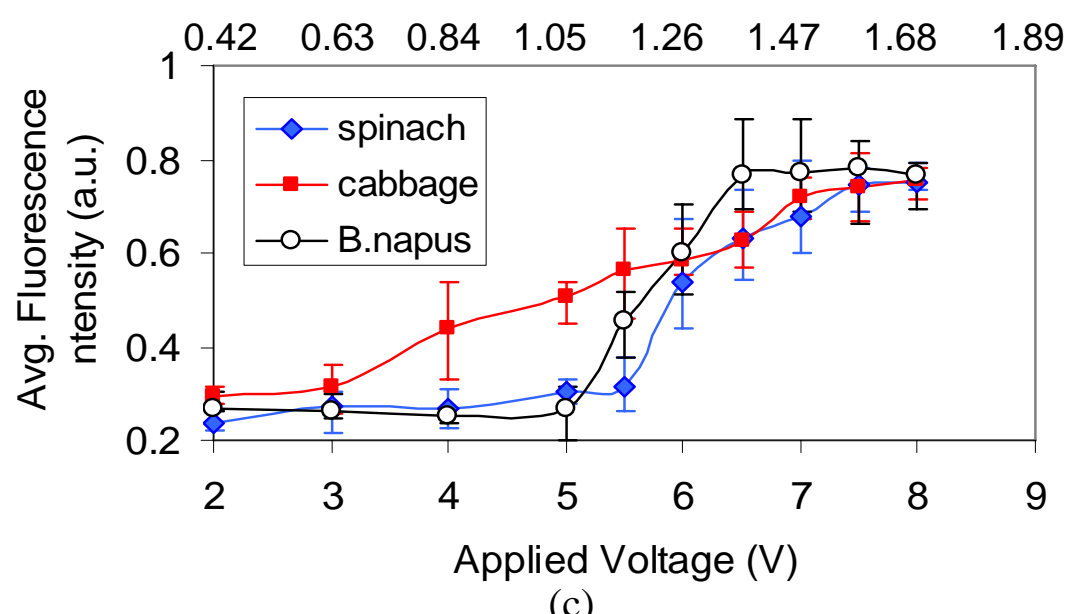

(c)

Fig. 8. Average fluorescent intensity as a function of applied voltage on 3 kinds of plant cells (Brassica pekinensis, spinach, and Brassica napus); (a) $t_{p}=2 \mathrm{~ms}$; (b) $t_{p}=4 \mathrm{~ms}$; (c) $t_{p}=6 \mathrm{~ms}$ (each data point the average from 10 cells). 


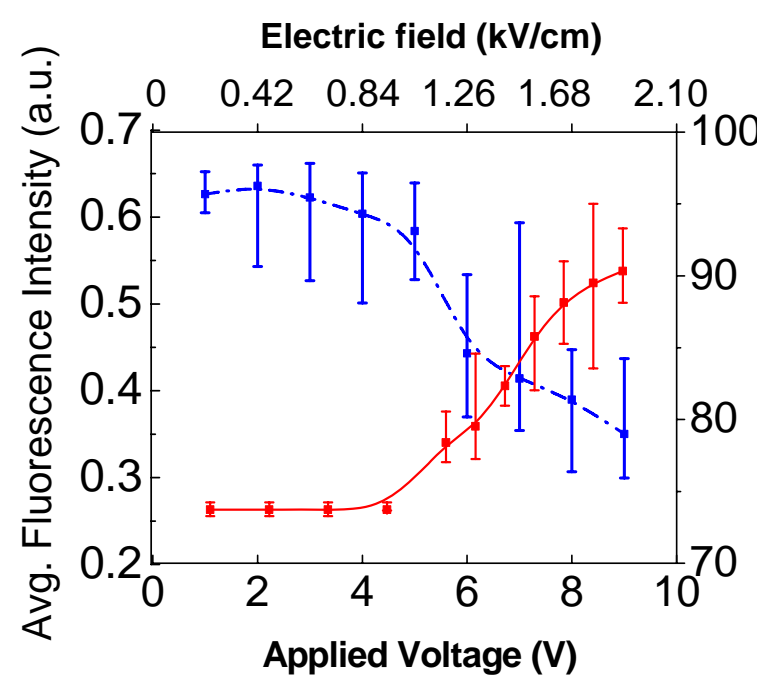

(a)

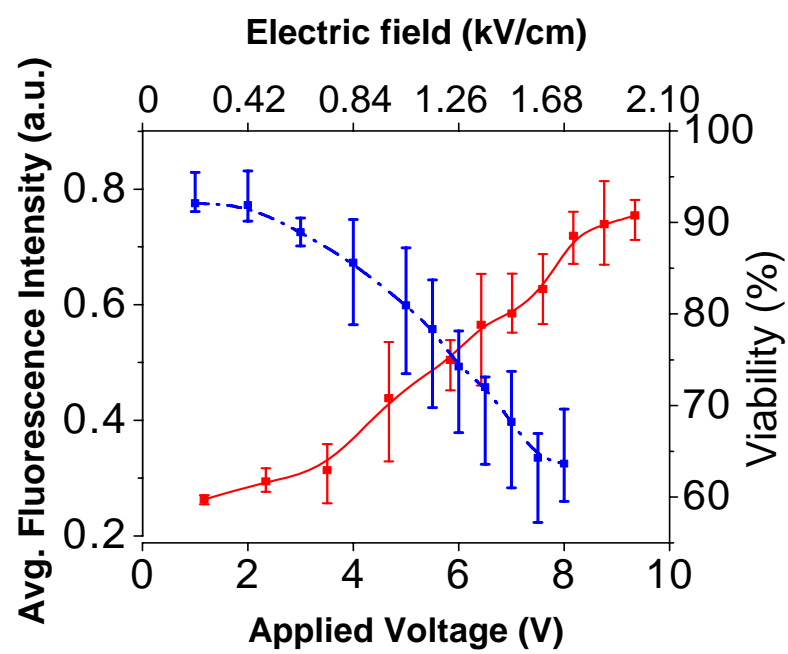

(c)

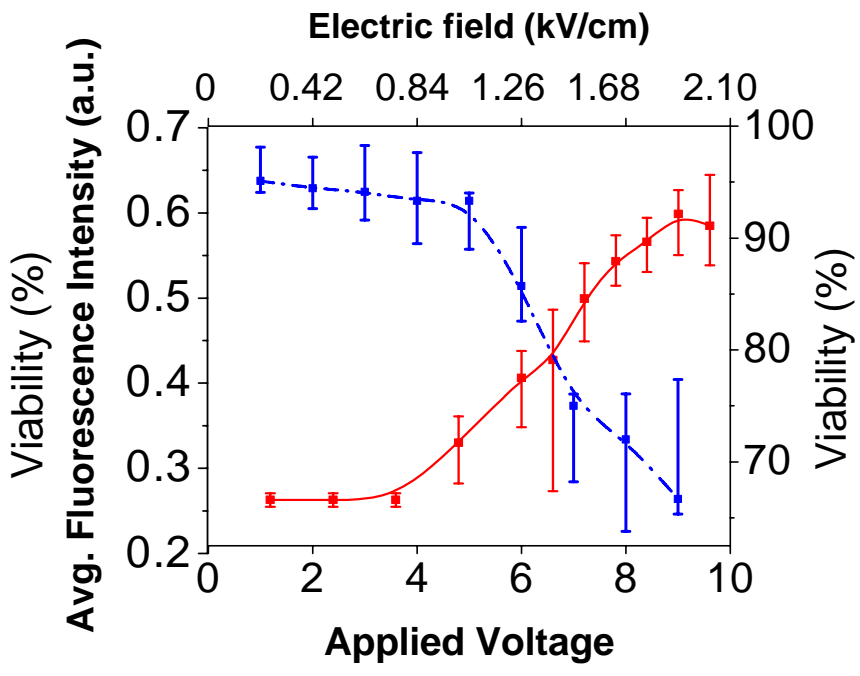

(b)

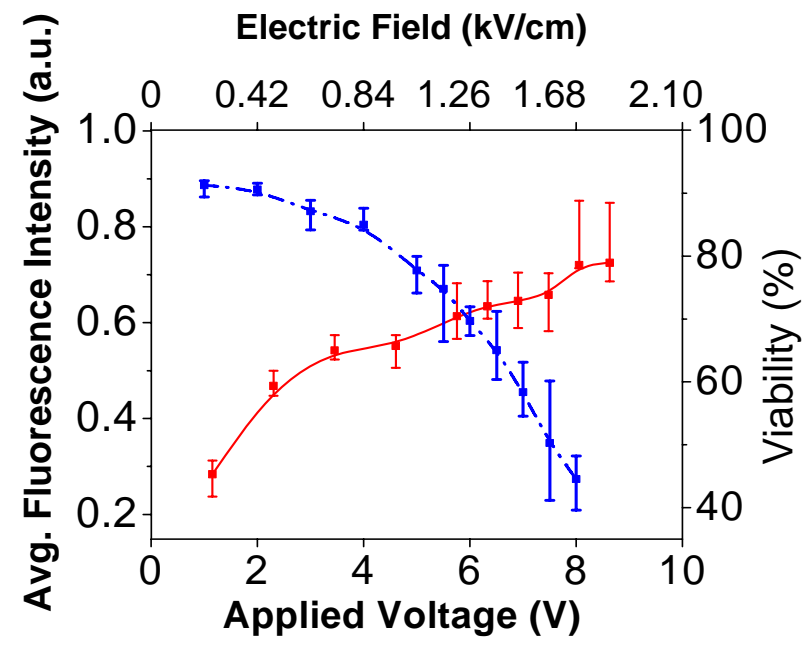

(d)

Fig. 9. Effect of applied voltage and pulse duration time on cabbage cell membrane permeability and viability (continuous line : Avg. fluorescence intensity, dash line: cell viability); (a) $t_{p}=2 \mathrm{~ms}$; (b) $t_{p}=4 \mathrm{~ms}$; (c) $t_{p}=6 \mathrm{~ms}$; (d) $t_{p}=8 \mathrm{~ms}$. 